\title{
THE RADIATION THERAPY IN OCCULT PRIMARY BREAST CANCER: CASE SERIES
}

Ana Flávia de Paula Guerra Campedelli', Nilceana Maya Aires Freitas', Thais Franco Simionatto1, Nathalya Ala Yagi', Jean Teixeira de Paiva', Carolina Martinelli Bezerril'1

${ }^{1}$ Hospital Araújo Jorge - Goiânia (GO), Brazil.

Objectives: Approximately $0.1-0.8 \%$ of all breast cancers is occult. The occult primary breast cancer (OPBC) is a breast cancer that first presents through regional nodal or distant disease without clinical or mammographic evidence of disease in the breast. Management of OPBC has been inconsistent and controversial since its initial description by Halsted in 1907, but the rarity of this form of breast cancer has made it difficult to standardize management. Furthermore, the natural history of OPBC remains unclear: most case series have reported outcomes that are better than those of non-occult breast cancers with similar nodal involvement, but a few have reported outcomes that are comparable or significantly worse. Here, we describe the management and outcomes of $\mathrm{OPBC}$ patients treated at a private and a public institution. Reviewing the management and outcome of $\mathrm{OPBC}$ patients we hope to elucidate both the benefits and disadvantages associated with a multidisciplinary treatment approach to OPBC. Methods: We described a four case series of women diagnosed with OPBC between 2015 and 2017 at Araujo Jorge Hospital and Cebrom Clinic, as well as the multimodal treatment received. All four patients were followed by a single observator. Case Report: The patients' ages ranged from 45 to 75 years, with a mean age of 60 years. The four patients presented with a palpable mass at the axillar area and all of them underwent level I and II axillary lymph node dissection pre radiotherapy. The pathologic results are invasive ductal carcinoma; 3 patients are grade 3 and 1 patient is grade 2; the imuno histologic results are 2 patients were luminous and 2 patients were triple negative; microscopic features revealed metastatic breast carcinoma in all of the four cases. All patients were submitted to adjuvant chemotherapy and then. 